\title{
BMJ
}

\section{Effectiveness of cervical screening with age: population based case-control study of prospectively recorded data}

\author{
Peter Sasieni, professor of biostatistics and cancer epidemiology Alejandra Castanon, epidemiologist Jack \\ Cuzick, John Snow professor of epidemiology
}

Cancer Research UK Centre for Epidemiology, Mathematics and Statistics, Wolfson Institute of Preventive Medicine, Bart's and The London School of Medicine, Queen Mary University of London, London EC1M 6BO

Correspondence to: $\mathrm{P}$ Sasieni p.sasieni@qmul.ac.uk

Cite this as: BMJ 2009;339:b2968 doi:10.1136/bmi.b2968

\section{ABSTRACT}

Objective To study the effect of cervical screening on incidence of cervical cancer as a function of age with particular focus on women screened under the age of 25 . Design Population based case-control study with prospectively recorded data on cervical screening. Setting Selected centres in the United Kingdom. Participants 4012 women aged 20-69 with invasive cancer diagnosed in participating centres and two controls per case individually matched on age and area of residence.

Main outcome measures Odds ratios for strength of association between cervical cancer and screening at particular ages.

Results There is no evidence that screening women aged 22-24 reduced the incidence of cervical cancer at ages 25 29 (odds ratio $1.11,95 \%$ confidence interval 0.83 to 1.50). Similar results were seen for cancers restricted to squamous carcinoma or FIGO (International Federation of Gynaecology and Obstetrics) stage IB or worse, but the numbers are insufficient to provide narrow confidence intervals. Screening was associated with a $60 \%$ reduction of cancers in women aged 40 , increasing to $80 \%$ at age 64. Screening was particularly effective in preventing advanced stage cancers.

Conclusions Cervical screening in women aged 20-24 has little or no impact on rates of invasive cervical cancer up to age 30 . Some uncertainly still exists regarding its impact on advanced stage tumours in women under age 30. By contrast, screening older women leads to a substantial reduction in incidence of and mortality from cervical cancer. These data should help policy makers balance the impact of screening on cancer rates against its harms, such as overtreatment of lesions with little invasive potential.

\section{INTRODUCTION}

Cervical screening is a complex process that requires careful analysis to determine the balance between its benefits and harms. For society it is important to show that screening will provide a net benefit at an affordable cost. These issues have been given prominence in the recent public controversy regarding screening in women aged 20-24.
Unfortunately policy makers are often forced to make decisions based on limited evidence. Such was the situation in 2003, when the screening programme in England was reorganised. One of those changes - to first invite women for cervical screening only once they reached the age of 25 (instead of between 20 and 24) - was and has remained controversial. ${ }^{1-6}$ The decision to change and standardise the age at first invitation was based, in part, on an earlier paper of ours, which showed that the relative reduction in frank invasive cervical cancer associated with screening was substantially less in women aged 20-34 than it was in older women. ${ }^{7}$

The existing literature is limited partly because causal inference from case-control studies is hampered by several biases and the possibility that factors other than those studied are driving the observed associations. Nevertheless, in the absence of randomised controlled trials addressing the particular question of interest, careful analysis of well designed observational studies provides the best evidence on which to modify existing screening programmes. The landmark meta-analysis from the International Agency for Research on Cancer (IARC) provided no details regarding the age dependence of the results, but stated that "age did not affect either the sensitivity of cytological screening or the distribution of the sojourn time of the disease. In particular, there was no evidence that younger women (under 35) were more at risk of developing fast growing tumours."8 We previously carried out a similar analysis on UK data in three age groups: 20-39, 40-54, and 5569 . We found that the reduction in risk three to five years after a normal smear result was greater in the older age groups. ${ }^{7}$ Our finding was confirmed by a smaller Italian study ${ }^{9}$ and, to a lesser extent, by a recent paper from Australia, ${ }^{10}$ but an important Swedish audit found no evidence of screening being less effective in young women. ${ }^{11}$

Here we studied how the association between cervical screening and a subsequent decrease in cervical cancer varies with age. We used a substantially enlarged dataset from the UK by estimating the odds ratios associated with screening in overlapping narrow age bands. Additionally, we focused on the age at 
which screening occurs rather than the age at which the cancers are diagnosed.

\section{METHODS}

Participants

Women diagnosed with cervical cancer were identified from histology laboratory records between January 1990 and April 2008. Local collaborators collected case-control data for a year at a time. Different centres collected cases over differing time periods depending on the availability of a collaborator. (See table on bmj.com for a breakdown of cases by year of diagnosis and region of residence within the UK.) Women with invasive (including micro-invasive) cervical cancer were classed as cases. Eligible controls were women who had ever been registered with a National Health Service (NHS) general practitioner (and had not subsequently died or emigrated). Such women have a record in the national cervical screening call/recall system. All controls were individually matched to cases on age and place of residence: one control had the same general practitioner as the case and a second had a different general practitioner but was within the same administrative area. Occasionally, only one control could be identified. The use of the same general practitioner provided a crude surrogate for socioeconomic status and ethnicity. We selected a control from a different general practitioner to avoid overmatching as screening uptake is closely related to the general practitioner's enthusiasm for cervical screening. We excluded cases not in the call/recall system at the time of diagnosis (because such women could not be selected as controls). Control selection was done blinded to the screening information and in most cases by random selection (with a computer program). Data were collected on all selected controls so there was no selection or participation bias. Data on screening histories were abstracted from routinely recorded cervical cytology records held on the call/recall system (and as such were not subject to recall bias). These records include all NHS (and many private) smear tests carried out in the UK since 1988. After local NHS staff linked screening data to cases and controls, the data were anonymised before being transferred to us for analysis. Details of the design have been published previously. ${ }^{712}$

After the publication of the paper in 2003 we found that 40 of the original cases had been duplicated (that is, the same woman was reported twice). As we have now removed the duplicates from the database the numbers of cases reported in the original article and the numbers of "previous" cases reported here are not the same. Figure 1 provides a breakdown of cases used throughout this paper.

\section{Statistical methods}

We used conditional logistic regression to estimate the association between having an adequate smear test taken in a particular three year age band (such as 22$24)$ with the incidence of cervical cancer in the subsequent five year band (such as 25-29). The association is

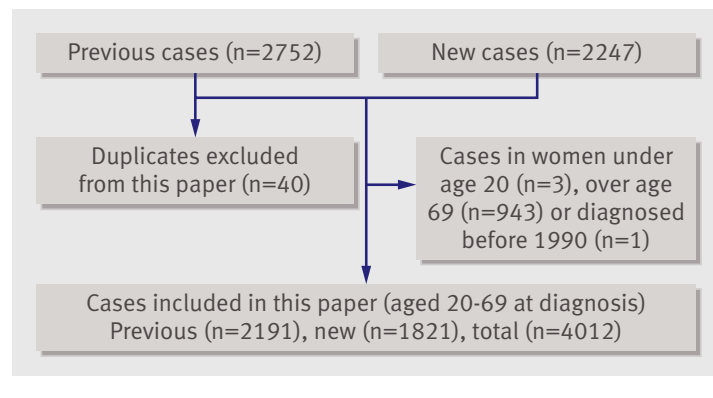

Fig 1 | Cases included in this paper

expressed as the odds ratio for developing cervical cancer in the next five year interval in those screened in a given (three year) age band compared with those not screened in that age band or in the two previous years. We repeated these analyses in overlapping age bands (that is, screening at 22-24, 23-25, 24-26, etc). All age bands are inclusive (that is, $22-24$ means $\geq 22$ and $<25$ ). See appendix I on bmj.com for further details, including the results of sensitivity analyses.

To ensure that age differences in effectiveness were not attributable to a different impact of screening on micro-invasive cancer or on adenocarcinoma, we looked at the effect of screening separately by stage and histology. Finally, we considered both all available data and only data obtained subsequent to our previous publication. ${ }^{7}$ The latter was done to examine possible trends associated with changes in screening policy and practice.

In an attempt to understand the reason for screening being less effective in young women we also looked, by age group, at the proportion of cases classified as screen detected, prevalent, interval, never screened or lapsed and "after an abnormal smear result". See appendix II on bmj.com for the intuitive description (as well the formal definition) of these categories.

Analyses were done in Stata 10 (StataCorp, College Station, TX).

\section{RESULTS}

Association between screening and cervical cancer at different ages

Figure 2 shows the main results, with selected details in table 1 . We included 4012 women with cervical cancer (any stage, including IA) diagnosed between 1990 and 2008 (including 1709 diagnosed since 2000) and 7889 controls. Figure 2 shows the odds ratio of cervical cancer in screened versus unscreened women as a function of age. At older ages the odds ratios are substantially below 1.0. They increase with decreasing age and are greater than 1.0 for those screened at age 20-22. The odds ratios relate to cancers diagnosed in a specific age band for women screened during a previous age band. For example, the estimated odds ratio of 0.26 plotted at a screening age band of 52-54 is to be interpreted as follows: the relative risk of having cervical cancer diagnosed at age $55-59$ is about 0.26 in women screened at age 52-54 compared with women not screened between ages 50 and 54 . These odds ratios vary from 0.18 to 0.36 in age bands from $40-42$ to $62-64$, 


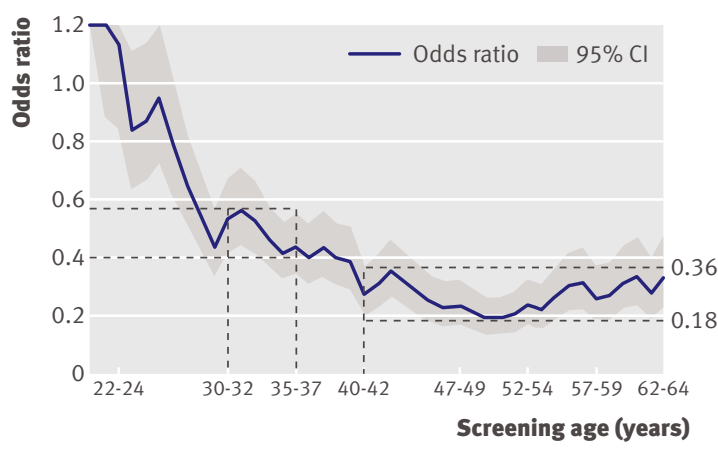

Fig 2 | Odds ratio for developing invasive cervical cancer stage IA or worse (in the next five year interval) in those screened in a given (three year) age band compared with those not screened in that age band (or in two previous years). Odds ratios plotted for overlapping age bands. Broken lines indicate risk of developing cervical cancer at ages 33-40 and 43-65. Odds ratios and confidence intervals are truncated at 1.2. Figure is based on 4012 cases (including 437 in women under age 30) and 7889 controls

respectively, corresponding to screening being associated with a reduction in the risk of cervical cancer over the subsequent five to eight years of between $64 \%$ and $82 \%$. In younger women the effect of screening is substantially and significantly less. Screening at ages 30-37 is associated with a reduction in the risk of cervical cancer over the next five years of between $43 \%$ and $60 \%$ (see broken lines on fig 2). The odds ratio for screening in the age band 22-24 is 1.11 (95\% confidence interval 0.83 to 1.50 ) (table 1). Similar estimates were obtained for screening at 20-22 and 21-23 (fig 2). Thus screening at ages 20-24 has no detectable impact on cervical cancer rates at ages 25-29.

\begin{tabular}{|c|c|c|c|}
\hline Age (years) at diagnosis of cancer & Cases (\%) & Controls (\%) & Odds ratio* $(95 \% \mathrm{Cl})$ \\
\hline \multicolumn{4}{|l|}{$25-29$} \\
\hline Screened age $22-24$ & $202(58)$ & 399 (57) & 1.11 (0.83 to 1.50$)$ \\
\hline Screened age $20-21$, but not $22-24$ & $46(13)$ & $70(10)$ & $1.51(0.95$ to 2.38$)$ \\
\hline Not screened aged $20-24$ & $103(29)$ & $226(33)$ & 1.00 \\
\hline Total & $351(100)$ & $695(100)$ & - \\
\hline \multicolumn{4}{|l|}{$35-39$} \\
\hline Screened age 32-34 & $346(53)$ & $842(66)$ & $0.55(0.44$ to 0.69$)$ \\
\hline Screened age $30-31$, but not $32-34$ & $88(14)$ & 144 (11) & $0.79(0.57$ to 1.1$)$ \\
\hline Not screened aged 30-34 & $214(33)$ & $288(23)$ & 1.00 \\
\hline Total & $648(100)$ & $1274(100)$ & - \\
\hline \multicolumn{4}{|l|}{ 45-49 } \\
\hline Screened age $42-44$ & $214(45)$ & $583(63)$ & $0.37(0.29$ to 0.48$)$ \\
\hline Screened age $40-41$, but not $42-44$ & $55(12)$ & $133(14)$ & $0.40(0.27$ to 0.58$)$ \\
\hline Not screened aged 40-44 & 203 (43) & $207(22)$ & 1.00 \\
\hline Total & $472(100)$ & $923(100)$ & - \\
\hline \multicolumn{4}{|l|}{$55-59$} \\
\hline Screened age 52-54 & $111(33)$ & $389(58)$ & $0.26(0.19$ to 0.36$)$ \\
\hline Screened age $50-51$, but not $52-54$ & $32(9)$ & 103 (15) & 0.27 (0.17 to 0.43$)$ \\
\hline Not screened aged 50-54 & $198(58)$ & $183(27)$ & 1.00 \\
\hline Total & $341(100)$ & $675(100)$ & - \\
\hline
\end{tabular}

BMJ | ONLINE FIRST | bmj.com
We also looked separately at the data on 1821 cases collected since our previous publication (figure not shown). The pattern was similar to that for all the data. In particular, odds ratios were still close to 1.0 for women screened at age 20-25.

\section{Association between screening at different ages and} cervical cancer by stage and histological type of cancer Figure 3 shows the sensitivity of these results to stage or histological type of cancer, or both. The overall patterns are similar to those in figure 2. In particular, when restricted to stage $\mathrm{IB}+$, the odds ratios are not significantly different from unity at young ages; as before they fall rapidly with increasing screening age from 25 to 34 years and then become fairly flat with a nadir around age 55 . The odds ratios are even lower for more advanced disease, showing that advanced cancer is particularly rare in screened women. The pattern for women with stage II or worse cancer is slightly different with a dip at age 22-24 and a second peak at age 2528 . There were only 38 cases of advanced cancer (stage II or worse) in women under age 30 so the estimated confidence intervals (fig $3 \mathrm{~d}$ ) are wide.

Despite there being over 350 women with cervical cancer (any stage) aged 25-29, there is no indication of any benefit of screening at age 22-24 (compared with those not screened at age 20-24) $(1.11,0.83$ to 1.50$)$ (table 1). Restriction of analysis to stage IB+ cervical cancer made little difference to the point estimate, but resulted in a wider confidence interval and allowed for the possibility of a greater effect $(1.03,0.63$ to 1.7$)$ (fig 3B). Further restriction to stage IB+ cervical cancer in women aged 25-27 at diagnosis (see appendix I on bmj.com) limited the analysis to 65 women and yielded an estimated odds ratio of 0.52 (0.23 to 1.2).

Benefit associated with being screened twice by age 26 It has been argued that screening begins to be fully effective only once a woman has been screened twice and that consequently women screened aged 20-24 and again at age 25 will have a greater benefit from screening after age 25 than will those who are first screened at age 25. To study this we compared women screened both at ages 20-22 and 23-25 with those first screened only aged 23-25. In an analysis of cancers diagnosed between ages 26.5 and 29.0 years restricted to women who were screened between ages 23 and 25 (inclusive) the odds ratio for stage IB+ cancer associated with also being screened between ages 20 and 22 (inclusive) was 0.90 (0.38 to 2.2). For all cancers (including stage IA) the odds ratio was 1.1 (0.62 to 2.0).

Screening classification of diagnoses in women before age 25 There were 73 cancers diagnosed in women at age 2024 (inclusive). Of these 73 women, five had had no previous smear tests, 32 were classified as screen detected (13 on their first screen and 19 on subsequent tests), 15 as interval cancers (last result was normal), and in 21 the diagnosis followed a history of abnormal 

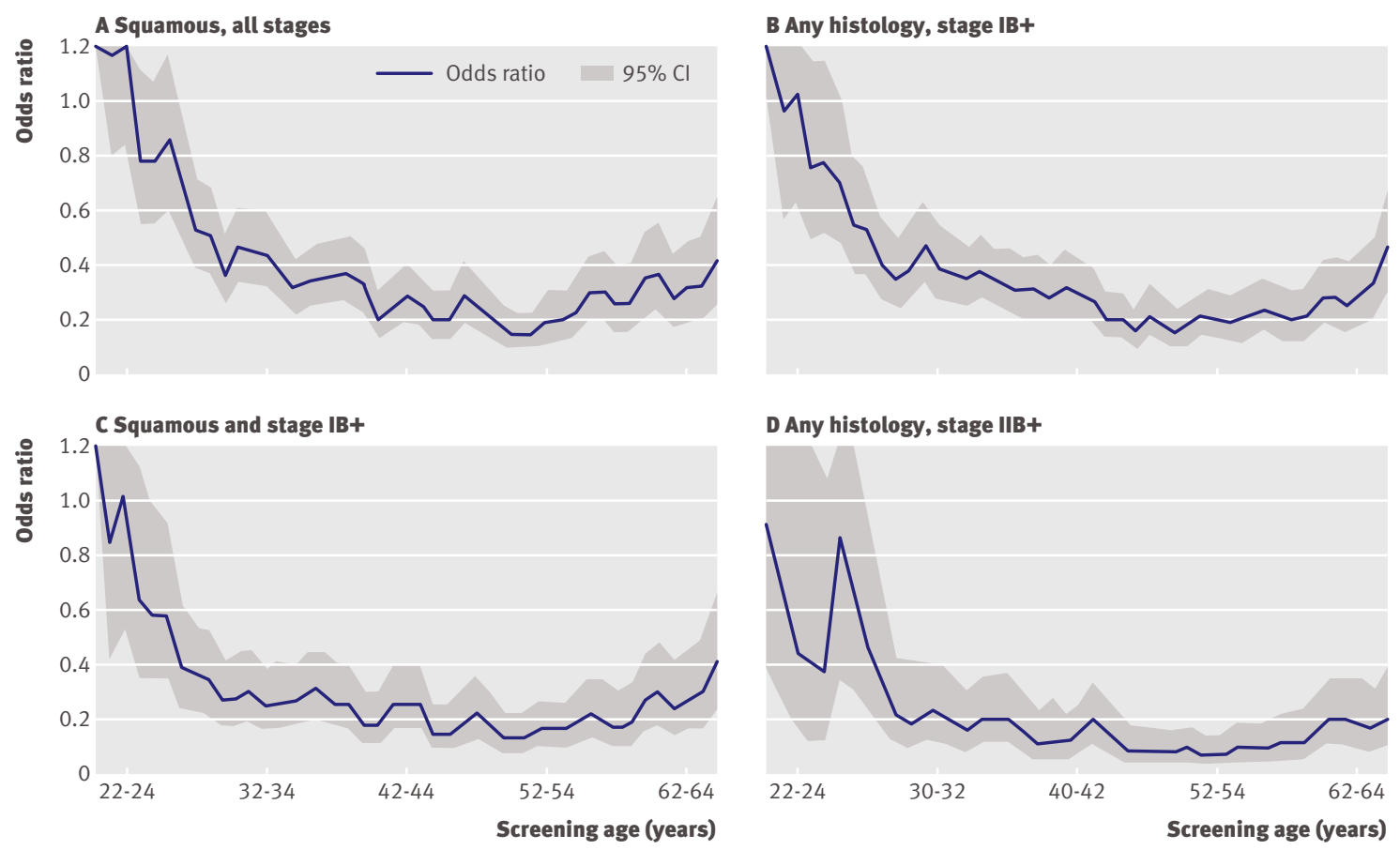

Fig 3 | Effect of stage and histology type on odds ratio of developing cervical cancer (in next five year interval) given screening in indicated age bands. Graph shows diagnosed cancer with five years of follow-up and compares those screened in the previous three years with those not screened in the previous five years. A: 2589 cases (303 in women under age 30 ) and 5122 controls; B: 2448 cases (172 in women under age 30) and 4821 controls; C: 1525 cases (107 in women under age 30$)$ and 3025 controls; D: 897 cases (38 in women under age 30 ) and 1764 controls

results (table 2). In these young women, 75\% of all cancers, $76 \%$ of stage IA cancers, and $81 \%$ of cancers stage IB or worse occurred despite screening. We consider cancers classified as interval, screen detected (previously screened), and "after an abnormal result" to have occurred despite screening.

Most screen detected (previously screened) cancers were micro-invasive (12 out of 19 with known stage), whereas most of the interval cancers were stage IB or worse (12 out of 15 with known stage) (Fisher's exact test $\mathrm{P}=0.017)$. Of the 18 stage $\mathrm{IB}+$ cancers in women with a previous normal screening history, $11(61 \%)$ occurred within 3.5 years of a negative smear test result. We applied the same classification of screening history to all women with cervical cancer stage IB or worse. Table 3 shows the results in 10 year age groups. The proportion of women with stage IB+ cancer who had

Table 2 |Screening history for women aged 20-24 at time of diagnosis. Figures are numbers (percentages) of women

\begin{tabular}{lcccc} 
& \multicolumn{4}{c}{ Stage } \\
\cline { 2 - 5 } Screening category & IA & IB + & Unknown & Total \\
Screen detected: & & & & \\
\hline First screen & $6(18)$ & $5(16)$ & $2(25)$ & $13(18)$ \\
\hline Previously screened & $12(36)$ & $6(19)$ & $1(13)$ & $19(26)$ \\
\hline Interval & $2(6)$ & $12(37)$ & $1(13)$ & $15(21)$ \\
\hline After abnormal cytology & $11(33)$ & $8(25)$ & $2(25)$ & $21(29)$ \\
\hline Never screened or lapsed & $2(6)$ & $1(3)$ & $2(25)$ & $5(7)$ \\
\hline Total & $33(100)$ & $32(100)$ & $8(100)$ & $73(100)$ \\
\hline
\end{tabular}

not been screened in the previous six years (never/ lapsed) increases with age. Compared with cases in women aged 40-69, women aged 20-29 with stage IB+ cancer were far less likely to be classified as "never screened."

\section{DISCUSSION}

This study confirms our previous findings that cervical screening in women aged 20-34 is less effective than in older women. By studying the effect of screening in smaller age groups, we have shown that the efficacy of screening decreases with decreasing age, even within the age range 20-34.

On average, participation in the UK cervical screening programme by a woman aged 35-64 reduces her risk of cervical cancer over the next five years by 60 $80 \%$ and her risk of advanced cervical cancer by about $90 \%$. The benefit of screening for women aged $25-34$ is more modest. Screening in women aged $20-24$ has little or no impact on the incidence of cervical cancer under the age of 30. This applied whether we looked at all cancers or restricted analysis to frankly invasive (that is, stage IB or worse) squamous carcinoma, or even to stage II or worse (fig 3). Because stage IB+ cancer is rare in young women, however, the confidence intervals are wide and our data do not rule out the possibility of screening in women aged 20-24 being effective in reducing stage IB+ cancer in women aged 25-27.

It has also been argued that women should have their first two smear tests close in time to minimise the 
Table $3 \mid$ Distribution of cancers stage IB or worse according to screening classification. Figures are numbers (percentages) of women

\begin{tabular}{|c|c|c|c|c|c|c|}
\hline \multirow[b]{2}{*}{ Screening category } & \multicolumn{6}{|c|}{ Age (years) at diagnosis } \\
\hline & $20-29$ & $30-39$ & $40-49$ & $50-59$ & $60-69$ & Total \\
\hline \multicolumn{7}{|l|}{ Screen detected: } \\
\hline First screen & $25(15)$ & $131(20)$ & $115(18)$ & $87(16)$ & 86 (19) & 444 (18) \\
\hline Previously screened & 33 (19) & $121(18)$ & $93(15)$ & $47(9)$ & $20(4)$ & 314 (13) \\
\hline Interval & $54(31)$ & $196(29)$ & $143(23)$ & $127(24)$ & $106(23)$ & $626(26)$ \\
\hline After abnormal cytology & $41(24)$ & $115(17)$ & $126(20)$ & $74(14)$ & $45(10)$ & $401(16)$ \\
\hline Never screened or lapsed & $19(11)$ & $102(15)$ & $148(24)$ & $193(37)$ & $203(44)$ & $665(27)$ \\
\hline Total & $172(100)$ & $665(100)$ & $625(100)$ & $528(100)$ & $460(100)$ & $2450(100)$ \\
\hline
\end{tabular}

impact of an initial false negative result. ${ }^{13}$ Our results provide no evidence that women screened aged 20-22 and then again at 23-25 are better protected than those screened only at age 23-25.

A careful review of the screening histories of women aged 20-24 with a diagnosis of cervical cancer suggests that few (if any) of the cancers occurred through a lack of screening. Indeed only five of these 73 women had not been screened previously.

\section{Strengths and weaknesses}

We used prospectively recorded screening data and selected controls at random, thus eliminating both recall bias and selection bias (data were obtained for all selected controls). We believe this design to be the most appropriate given that a randomised controlled trial was not possible. Other papers have analysed trends in incidence of cancer (or mortality, or both) before and after the introduction of screening to estimate the impact of screening in the population. ${ }^{14-1 / 2}$ Such analyses rely on comparison of observed rates with estimates of what would have happened in the absence of screening; they are subject to trends in other factors such as the quality of the cancer registry data. For women aged 20-29, who have been offered screening from the age of 20 , it is not possible to reliably estimate what their rates would have been in the absence of screening.

It is always possible to criticise observational studies as women who attend screening might differ from those who do not so that any observed effect might not be causal. For the observed difference in the benefit of screening at different ages to be caused by confounding, however, there would have to be differences in the way confounders affect the results at different ages. We know of no evidence to support such an interaction and suggest that differential benefits with age are not caused by confounding but reflect the true effects of screening. We think there are few biases in this analysis and are comfortable in viewing the associations as causal and using the term "protection offered by screening" to describe the odds ratios.

This large study of the impact of cervical screening on invasive cervical cancer contains more cases than all other studies with detailed screening information combined. Some of the data presented have been published previously, but over $45 \%$ are new. The results of analyses limited to the new data are qualitatively similar to those using all the data, suggesting that there have been no changes in the impact of screening in young women on rates of cervical cancer despite improvements in the quality of screening in the UK.

The new analysis considers the association between screening in one age group (for example, 25-29) and cervical cancer in the subsequent five years (at ages 30-34 for this example). With this approach, the exposure is close to the usual definition of screening coverage used by many screening programmes: the proportion of eligible women screened in the past five (or three) years. Furthermore, this approach more closely reflects what one could estimate by prospectively following a cohort of women. With a coverage interval of three years and a follow-up of five years, however, it could be as much as eight years between the last screen and a diagnosis of cancer. Thus the benefit of regular three (or five) yearly screening could be considerably greater than that implied by this model. Nevertheless, the approach does attempt to measure the protection achieved with screening (from the treatment initiated by a positive smear result) rather than the low risk periods associated with a negative smear result.

\section{Comparison with other studies}

Much of the earlier literature aiming to study the protection afforded by cervical screening did not consider the possibility of different levels of protection depending on age. An important recent paper reported on the results of an audit of cervical cancer in Sweden. ${ }^{11}$ The odds ratios in that paper are similar for all age groups with an odds ratio of $0.42(1 / 2.37)$ for the effect of three yearly screening on incidence of cancer at ages 21-29. It is important to consider the methodological differences between the analyses when interpreting these results. They consider a woman (aged 20-52) to have been screened if she had a smear test between 3.5 years and 6 months before (the date of the case's) diagnosis. They include stage IA cancers, most of which will have been screen detected, as well as screen detected stage IB cancer. Consider a screen detected cancer in a woman who has two smear tests 3.5 years apart. If the smear test that led to diagnosis is within six months of diagnosis she will be classed as unscreened. A control 
woman who is screened every 3.5 years will have a chance of $86 \%(3.0 / 3.5)$ of having had a smear test in the particular interval width of three years. Thus the inclusion of screen detected cases introduces a considerable bias in favour of screening. As the proportion of cancers that are stage IA or screen detected stage IB is greater in young women, the bias is particularly strong in young women. To illustrate the point, the same analysis applied to women aged 20-29 in our study yields an odds ratio of 0.46 (0.38 to 0.56 ).

A case-control study in New South Wales, Australia, found that screening every two years seemed to be more protective in women over the age of 30 than in those aged $20-29 .{ }^{10}$ The more favourable results for screening in women aged 20-29 in that study could be because their controls were selected from women who had been for screening (albeit possibly only after the date of diagnosis of the case).

\section{Interpretation of the results}

As we designed the study to eliminate most of the biases that affect case-control studies, our observed associations are almost certainly either causal or the result of confounding. The heterogeneity of association in different age bands within the same study argues strongly that these effects are real. There might be biological reasons for cervical screening working better in older women. Undoubtedly the specificity of screening is less in younger women because human papillomavirus (HPV) infections are so much more common. This does not, however, explain why the sensitivity of screening should be less. We favour the explanation that, by necessity, a cancer in a woman aged 25 (infected at, say, 15 years) will have progressed from HPV infection through cervical intraepithelial neoplasia grade III to cancer faster than in a woman aged 55 (infected at perhaps 25). This means that the opportunities for detecting the small proportion of cervical intraepithelial neoplasia grade III in women in their early 20 s that will progress to cancer within at most a few years are small. It is an extreme example of length bias: most cases of cervical intraepithelial neoplasia grade III detected will be slow growing and could safely be left for several years; but the rare cases that are progressing rapidly will probably be missed.

It has been argued that screening from age 20 could prevent more cancers in women aged 25-34 than screening from age 25 . Our study has little power to detect such an effect. Policy decisions should be based on balancing the benefits and harms of screening and the need to take into account the underlying risk of cervical cancer at different ages. Such an analysis is beyond the scope of this paper. We have provided more accurate estimates of the benefits of cervical screening in different age groups, which should aid policy makers in making their decisions. As screening undoubtedly leads to the detection of many cases of stage IA cancer in women aged 20-24, one might think it must be doing good by preventing more advanced cancers. If this were the case, we should have found that screening at ages 20-24 leads to a reduction in stage IB+ cancers. That we found no such reduction suggests that most of the stage IA cancers detected by screening women aged under age 25 would still be stage IA at ages 25-26 and could be picked up by screening at age 25 without adverse consequences.

This study is based on cancers diagnosed in the UK between 1990 and 2008 and smears taken within the UK from 1988 to 2008. Cervical cancer rates in women in their 20s have been relatively high compared with those in other countries and abnormal cytology results have been more common in those aged 20-34 than in older women throughout this period. ${ }^{1819}$ We believe that the standard of smear taking, cytology reading, and fail safe procedures for cervical screening in this study have mostly been high. Since the early-mid 1990s the UK screening programmes have put great emphasis on quality assurance, and there is evidence that by the late 1990s, UK cytology was as good as (or better than) anywhere in the world. ${ }^{20}$ Thus our finding that cervical screening in women aged $20-24$ has at best a modest effect on the incidence of cervical cancer at ages 25-29 is almost certainly generalisable to other countries. We have no reason to believe that it would be substantially more effective elsewhere.

Any decision on when to start screening women will have to weigh up benefits and harms and might depend on the local status quo. In a setting where screening is offered to women aged 20-24 policy makers might decide to continue this policy as we have not shown that the harms exceed the benefits. By contrast, where screening is not offered to women aged 20-24, the lack of evidence of any benefit from screening in this age group dictates that the policy should not change.

\section{Unanswered questions and future research}

Our study does not consider the harms of screening at different ages nor do we take into account the absolute rate of cervical cancer in screened and unscreened women of different ages. Such a synthesis of research is necessary for rational policy making. Undoubtedly, however, decision making will be complicated because of the uncertainty in many of the estimates of harm and benefit. As we have seen, the confidence intervals for the impact of screening at ages 20-24 on stage II+ cervical cancer are extremely wide. We have not even attempted to estimate the added impact of starting screening five years earlier on cancer at ages 30-44.

The most common harms of screening are the anxiety caused by abnormal test results and the trauma of treating cervical intraepithelial neoplasia that would never have progressed to cancer. These can be easily estimated. Treatment might be associated with premature delivery during subsequent pregnancies. If the association observed in several studies ${ }^{2122}$ is causal then screening might do serious harm, but the association might simply be because of confounding. These issues require careful study.

The question of screening women aged 20-24 will decrease in importance as the cohort of women vaccinated against HPV types 16 and 18 reach their 20s. If it 


\section{WHAT IS ALREADY KNOWN ON THIS TOPIC}

Cervical screening has had a substantial impact on the incidence and mortality of cervical cancer in many developed countries

Most of the benefit from screening comes from the prevention of cervical cancer, but it can also lead to downstaging

The relative protection against cervical cancer might be less at ages 20-34 than in older women

\section{WHAT THIS STUDY ADDS}

Cervical screening in women aged 20-24 is substantially less effective in preventing cancer (and in preventing advanced stage tumours) than is screening in older women

The new methods for the analysis of case-control studies of screening avoid some of the biases associated with previously used statistical methods

is questionable whether screening is worth while in unvaccinated women aged 20-24, there can be no doubt that the risk of cancer in women aged under 25 who are vaccinated before exposure to HPV will be low enough to make screening at such an age unjustifiable.

We thank all those who have helped with the design and conduct of this study and acknowledge the participation of millions of women and nameless healthcare professionals in the cervical screening programmes in the UK.

Working group members: Joanna Adams, Alejandra Castanon, Jack Cuzick, Elaine Farmery, Hillary Fielder, Muir Gray, David Mesher, Julietta Patnick, and Peter Sasieni.

Local collaborators: C Camilleri-Ferrante and A Thompson (East Anglia); P Grey and M J Platt (Macclesfield and Warrington); D Haran (Chester and Wirral); F Fowler (Southend); S Chatterton (Oxfordshire, Northants, Buckinghamshire, Berkshire); S Butterworth, M Vaille and J Underdown (Maidstone); R Swann (Medway); L Robinson (south west Surrey, west Surrey and north east Hampshire); A Burtenshaw (Mid Downs); S Samarsinghe (Kingston and Richmond); C Furlong (Enfield and Haringey); C Singleton (N Derbyshire); K Boyd (East Dorset); A Herbert and $C$ Breen (Southampton and south west Hampshire); E Farmery (Wiltshire and Bath); L Daborn and K Jaber (west Dorset); J Grainger (Shropshire); G D H Thomas (Calderdale); W Young (Humberside); S Jennings (Leicestershire); F Boer (Brighton and Hove); RJ Fitzmaurice (Huddersfield); Y Burlay and H Belza (Grimsby); S Barraclough and H Belza (Scunthorpe). I Duncan and K A Hussein (Dundee and Angus); L Reay (Argyll and Clyde); L Caughley (Northern Ireland); S Burgess (Clwyd); DC Watkins (Gwent); Helen Beer (Cervical Screening Wales); Quality Assurance Reference Centre (QARC) in north west England and the QARC in east England.

Contributors: PS participated in the design and establishment of the study, collation of data, design of the database, and analysis of the data. AC participated in the collation and analysis of the data. JC participated in the design and establishment of the study. All authors wrote the paper and saw and approved the final version. PS is guarantor. Funding: This work was supported by Cancer Research UK (C8162/A6127 and C8162/A9481) and previously by the NHS cervical screening programme. Neither organisation had any input in the analysis or interpretation of the data or the writing of the paper.

Competing interests: None declared.

Ethical approval: Not required. Permission to link cervical screening and histology (cancer registration) data has been granted by the Patient Information Advisory Group (PIAG).
1 Fiander AN. Cenvical screening in young women aged 20-24 years. J Fam Plann Reprod Health Care. 2008;34:19.

2 Nair MS, Bhandari HM, Nordin AJ. Cervical cancer in women aged less than 25: east Kent experience. J Obstet Gynaecol 2007;27:706-8.

3 Rieck GC, Tristram A, Hauke A, Fielder H, Fiander AN. Cervica screening in 20-24-year olds. J Med Screen 2006;13:64-71.

4 Crocetti E, Battisti L, Betta A, Palma PD, Paci E, Piffer S, et al. The cytological screening turned out effective also for adenocarcinoma: a population-based case-control study in Trento, Italy. Eur J Cancer Prev 2007;16:564-7.

5 Herbert A, Holdsworth G, Kubba AA. Cervical screening: why young women should be encouraged to be screened. J Fam Plann Reprod Health Care 2008;34:21-5.

6 Bano F, Kolhe S, Zamblera D, Jolaoso A, Folayan O, Page L, et al. Cervical screening in under 25s: a high-risk young population. Eur J Obstet Gynecol Reprod Biol 2008;1389:86-9.

7 Sasieni P, Adams J, Cuzick J. Benefit of cervical screening at different ages: evidence from the UK audit of screening histories. Br J Cancer 2003;89:88-93.

8 IARC Working Group on Cervical Cancer Screening. Screening for squamous cervical cancer-the duration of low risk following negative results in cervical cytology test: introduction. IARC Sci Pub 1986;76:15-24

9 Zappa M, Visioli CB, Ciatto S, lossa A, Paci E, Sasieni P. Lower protection of cytological screening for adenocarcinomas and shorter protection for younger women: the results of a case-control study in Florence. Br J Cancer 2004;90:1784-6.

10 Yang B, Morrell S, Zuo Y, Roder D, Tracey E, Jelfs P. A case-control study of the protective benefit of cervical screening against invasive cenvical cancer in NSW women. Cancer Causes Control 2008;19:569-76.

11 Andrae B, Kemetli L, Sparen P, Silfverdal L, Strander B, Ryd W, et al. Screening-preventable cervical cancer risks: evidence from a nationwide audit in Sweden. I Natl Cancer Inst 2008;100:622-9.

12 Sasieni PD, Cuzick J, Lynch-Farmery E. Estimating the efficacy of screening by auditing smear histories of women with and without cenvical cancer. The National Co-ordinating Network for Cervical Screening Working Group. Br J Cancer 1996;73:1001-5.

13 Saslow D, Runowicz CD, Solomon D, Moscicki AB, Smith RA, Eyre HJ, et al. American Cancer Society guideline for the early detection of cervical neoplasia and cancer. CA Cancer / Clin 2002;52:342-62.

14 Anttila A, Pukkala E, Soderman B, Kallio M, Nieminen P, Hakama M Effect of organised screening on cervical cancer incidence and mortality in Finland, 1963-1995: recent increase in cervical cancer incidence. Int J Cancer 1999;83:59-65.

15 Bray F, Loos AH, McCarron P, Weiderpass E, Arbyn M, Moller H, et al. Trends in cervical squamous cell carcinoma incidence in 13 European countries: changing risk and the effects of screening. Cancer Epidemiol Biomarkers Prev 2005;14:677-86.

16 Pettersson F, Bjorkhom E, Naslund I. Evaluation of screening for cervical cancer in Sweden: trends in incidence and mortality 1958. 1980. Int J Epidemiol 1985;14:521-7.

17 Sigurdsson K, Sigvaldason H. Is it rational to start population-based cervical cancer screening at or soon after age 20? Analysis of time trends in preinvasive and invasive diseases. Eur J Cancer 2007;43:769-74.

18 Curado MP, Edwards B, Shin HR, Storm H, Ferlay IMH, et al. Cancer incidence in five continents Vol IX. Lyon: IARC Scientific Publications, 2007.

19 Health and Social Care Information Centre. Cervical screening programme, England: 2005-06. Leeds: Information Centre, 2006.

20 Cuzick J, Clavel C, Petry KU, Meijer CJ, Hoyer H, Ratnam S, et al. Overview of the European and North American studies on HPV testing in primary cervical cancer screening. Int / Cancer 2006;119:1095-101.

21 Kyrgiou M, Koliopoulos G, Martin-Hirsch PL, Arbyn M, Prendiville W, Paraskevaidis E. Obstetric Outcome after conservative treatment for intraepithelial or early invasive cervical lesions: systematic review and meta-analysis. Lancet 2006;367:489-98.

22 Jakobsson M, Gissler M, Sainio S, Paavonen J, Tapper AM. Preterm delivery after surgical treatment for cervical intraepithelial neoplasia. Obstet Gynecol 2007;109:309-13.

Accepted: 16 July 2009 